\title{
GIS for Achieving Sustainable Development of Gas Industry
}

\author{
Alexander S. Kazak ${ }^{*}$ and Maxim V. Komarov
}

NIIGazeconomica LLC, Staraya Basmannaya st., 20-8, Moscow, 105066 Russia

\begin{abstract}
The main goal of gas industry development strategy is to find and justify the optimum balance between gas production and consumption, ensure harmonious and synchronous advancement of all system developing sectors. This task imposes certain requirements on methods of storing and visualizing information in gas industry, its past, current status and future development. For this purpose, Gazprom VNIIGAZ-NIIGazeconomica LLC has developed its own version of geo-information system, which allows to efficiently structure, analyze, revise and visualize all information on the long-term development programs of gas industry by means of synthesizing tabulated and cartographic data.
\end{abstract}

Keywords: GIS, Master Plan, gas industry, cartographic data, sustainability.

\section{INTRODUCTION}

Development of long-term strategies for gas industry normally requires synthesis and joint analysis of information in such subsystems as geology and geological survey, field development, gas consumption, transmission and storage, as well as assessment of potential environmental, economic and infrastructural impacts. A common standard was to present hard copy reports of such information for quite a long time (Connolly \& Begg 2003).

Today we observe gradual transition from hard copy reports to information systems, where numerous multi-purpose geo-information systems (GIS) are developed capable of storing and visualizing cartographic data with tabulated information (Zhurkin \& Shaitura 2009). A de facto standard for many companies has become the ArcGIS system created by the Environmental Systems Research Institute (ESRI). However, its main disadvantage relevant to the objectives described is the system's multi-purpose design: ArcGIS does not deal with any specific characteristics whatsoever of the data required for gas projects and does not provide necessary search and query tools. To make its function this way, automation of ArcGIS is required which can be fulfilled by a team of experienced software developers within a few months, who can make the system operate on a particular set of data. However, to ensure that the data are constantly modified, updated and added, the programmers would each time have to revise the complete automation model, which is time-consuming and labor-intensive. Therefore, an internal specialized geo-information system was needed to serve as a practical working tool capable of visualizing the overall data and facilitating information search, modification, scaling and updating.

*Address correspondence to this author at NIIgazeconomica: Prof., Dr (tech) Alexander Kazak, 1-st Deputy Director, Institute of economics \& management of gas industry LLC, Gazprom (NIIgazeconomica), Staraya Basmannaya st., 20-8, Moscow, 105066 Russia; Tel: +7 499 265-24-20; Fax: +7 499 267-30-76; E-mail: a.kazak@econom.gazprom.ru
GIS STRUCTURE AND FUNCTIONS FOR GAS INDUSTRY SUSTAINABLE DEVELOPMENT: MASTER PLAN REQUIREMENTS

Being responsible for Master Plan development of gas industry both in the whole area of Russia and in its Polar region, Gazprom VNIIGAZ-NIIGazeconomica LLC have developed the first version of this geo-information system (Kazak et al. 2010). The key system requirements have the ability to efficiently store, visualize and provide functional tools for search and analysis of information not only on retrospective development of the Bolivian gas industry but on the forecasts of its future according to various scenarios. The task was complicated as each scenario was specific in terms of mineral resource base development, gas demand, production and building up capacities in transmission and processing sectors. This inevitably led to variations in environmental, economic and other parameters. The new GIS made it possible to structure and visualize all the multidisciplinary and diverse information on the specified aspects of the Master Development Scheme.

When GIS project was launched the developers decided to save all cartographic and reporting information in the database (Connoly \& Begg 2003; Kline 2006). The final database distributed together with the software allows to perform a concluding demonstration of works on any computer without any preliminary preparation. The system involves separation of user rights for access to information and functional tools. It also permits to establish a bi-directional connection between cartographic and reporting data on all the facilities, subsystems and areas.

The first version of the system stored all the cartographic information in shapefile format, and gas industry subsystems data in MS Excel format. This significantly simplified preparation and processing of the initial tabulated and cartographic data.

Aside from the tools available to users for viewing and working with information, the administrative interface provides basic GIS mapping functionality, i.e. simple map 
modifications can be performed directly from the software interface without recourse to other products.

The basic information unit of the software is a reporting document that may contain information in the form of tables and figures (diagrams, graphs, maps, etc.), and their number is unlimited. To facilitate analysis the reporting field is divided into two areas: tables are separated from figures and, in viewing a particular table, a user can flip through diagrams reflecting different aspects of the data presented. The areas containing tables and figures are easily customized and they can be changed in size or minimized. Thus, excess information that impedes browsing can be hidden. A reporting document can be exported to an MS Excel file or other formats for further work or publication. The original table format is preserved and the figures are placed under the tables.

In addition to reporting documents, the user may get access to attributive data on the layers and facilities specified in the map. The access may be given via a list of layers or a selected facility. This attributive information is held in $\mathrm{dbf}$ tables/shapefile format and can be used to display captions, search the required areas, facilities and geographic coordinates. For the sake of convenient navigation, various filters may be used to create data clusters to be saved and applied in further work.

In the course of project creation, a list of available reporting documents is formed in the data base with the main menu reflecting the data hierarchy to provide access to documents. A click on the corresponding menu item opens a report in a new window. When several reports are open, they are arranged in the window as tabs thus allowing to easily switching from one to another. This mode is especially convenient when two computer monitors are used, one - to display a map and the other one - to show the reporting documents.

Moreover, any facility depicted in the map (or a few facilities at a time) can be matched with the corresponding data retrieved from the database. This allows users to open a corresponding report after the appropriate facility is selected and systemize and facilitate search for detailed information on any facility of gas industry subsystem including infrastructure projects.

If a map segment is selected which contains several facilities that can be attributed to a reporting document the needed report may be found in a context menu. Documents there are arranged into cluster tree structures relevant to the facilities located in the selected area. Therefore, the information space gets restricted to a required area.

Any data contained in the system may be visualized in the form of summary tables containing complete information on a particular aspect. If the reports do not provide the required data, they can be obtained from a summary table. To do this, the user can, by sending commands, (as a subset of SQL language [Klein, 2006]) filter the necessary data, add aggregated lines and columns (average, total, mathematic expectation, etc.) and draw diagrams. All assembled the information may be saved as a stand-alone reporting document, stored in the data base and added to the existing hierarchy.

If the required data are available, it takes just a few hours to set up a GIS project and format it in the system. This also requires no high qualifications of the operator. Data modification can be performed in a flash since specialized GIS does not require improvement or automation to meet the requirements of the gas industry specialists.

\section{CONCLUSION}

The software interface has a modern design and stands alone among many specialized programs that focus on functionality but ignore convenience and visual aesthetics. Here fair attention is given to functionality and interface design. The main panel represents an MS Office 2007-style line instead of a conventional menu and toolbar. It proved to be quite effective over the past three years and has been adopted by many large developers.

The software can be rapidly deployed, with the installer automatically setting up the required components, creating the data base and adjusting the program. No special skills are needed to install the software. The GIS project has been tested on Windows XP, Windows Vista and Windows 7 in various language environments and on different equipment thus ensuring that no operational problems should occur in the future.

Application of this system enables specialists to perform a more efficient expert analysis which is crucial to the future success of the entire gas industry.

\section{CONFLICT OF INTEREST}

The authors confirm that this article content has no conflicts of interest.

\section{ACKNOWLEDGEMENTS}

The authors express their deep appreciation and gratitude to D. Ratner, Institute of economics and management of gas industry, NIIGasecomonica, for the consultation and active participation during result discussions.

\section{REFERENCES}

Connolly, Th \& Begg, C (2003) Database Systems:Practical Approach to Design, Implementation, and Management, Third Edition, Williams, Moscow.

Kazak, AS, Rusakova, VV, Gitman, IS \& Kudryavtsev, IB (2010) Geoinformation systems and gas industry development strategy. Science and Technology in Gas Industry, 2 (42), 40-4.

Kline, K (2006) «SQL», KUDITS-OBRAZ Publishers, Moscow.

Zhurkin, IG \& Shaitura, SV (2009) Geo-Information Systems, KUDITSPRESS, Moscow. 